\title{
Sepse - Conduta baseada no Protocolo Clínico utilizado na Unimed Recife
}

\author{
Sepsis - Conduct based on the Clinical Protocol used at Unimed Recife
}

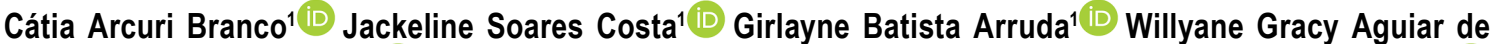
Andrade Gomes de Souza' (iD) Alex Maurício Garcia Santos ${ }^{2,3}$ (D) Fabiana Gonzaga Torreão dos Santos ${ }^{2,3}$ (iD) Jeniffer Cristina Rocha Cruz ${ }^{2}$ (D) Lais Emanoelle de Souza Cabral ${ }^{2}$ (D) Adriana Cesário Lopes ${ }^{3}$ (iD Orlando José de Lima Nascimento ${ }^{3}$ (D) Martha Maria Romeiro Figueiroa F. Fonseca ${ }^{1}$ (iD
\end{abstract}

${ }^{1} \mathrm{H} o s p i t a l$ Unimed Recife III, ${ }^{2} \mathrm{Hospital}$ Geral Materno-Infantil, ${ }^{3}$ Hospital Unimed Recife I, Recife, Pernambuco, Brasil

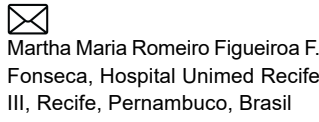

martharomeiro@globo.com

Este artigo foi editado por Marcelo Moraes Valença Juliana Ramos de Andrade

Palavras-chave:

Sepse

Protocolo clínico

Tratamento

Diagnóstico

Critérios

Keywords:

Sepsis

Clinical protoco

Treatment

Diagnosis

Criteria

\section{Resumo}

Pacientes com suspeita de sepse, com base no critério SIRS (systemic inflammatory response syndrome), são bastante identificados na emergência e responsáveis por 30\% dos pacientes admitidos na UTI. Triagem precisa, reconhecimento rápido, reanimação precoce, início imediato de antibióticos e erradicação da fonte de infecção são os principais componentes no atendimento de sepse de qualidade. Sabemos que sepse é uma causa frequente de mortalidade hospitalar e que sequelas, além de um maior risco de morte, também, estarão presente nos pacientes após a alta.

A implantação de protocolos clínicos relacionados com sepse desde a primeira publicação, em 2001, com terapia direcionada por objetivo inicial, provou ter um impacto marcante na letalidade em nossos pacientes. Com o objetivo de atualizar o protocolo, utilizando maior acurácia e melhor gerenciamento de sepse, ademais da implementação do protocolo de sepse, estamos incluindo as definições mais recentes e comentando o uso de marcadores clínicos utilizados para suspeita e diagnóstico mais preciso de sepse.

\section{Abstract}

Patients with suspected sepsis based on the systemic inflammatory response criterion syndrome (SIRS) are quite identified in the emergency room and are responsible for $30 \%$ of the patients admitted to ICU. Accurate screening, rapid recognition, early resuscitation and antibiotics administration, and eradication of the source of infection are the main components in quality sepsis care. We know that in addition to the frequent association between the causes of hospital mortality, we also recognize that sequelae and an increased risk of death will also be present in post-discharge patients. The implementation of sepsis protocols since the first publication in 2001 of the results of the initial objective-directed therapy trial has proven to have a marked impact on mortality. In order to update and expand the protocol using greater accuracy and better sepsis management, we are now performing an implementation of the sepsis clinical protocol. Updating definitions and using the latest clinical markers used for suspected sepsis. 


\section{Introdução}

epse é uma condição clínica considerada como doença emergente com grande impacto no sistema de saúde pelo alto risco de morte. A incidência e mortalidade relacionadas à sepse têm crescido, devido ao aumento da expectativa de vida e manejo mais efetivo de condições crônicas, doenças neoplásicas, e uso mais frequente de imunossupressores. ${ }^{1,2}$

O retardo na identificação precoce dessa condição corrobora com o início tardio de um tratamento adequado imediato e, como consequência, se associa com desfechos desfavoráveis (e.g., sequelas e mortes). ${ }^{2}$ Sepse é a principal causa de morte em pacientes com infecção. Dessa maneira, a sepse pode ser definida como qualquer infecção que acarrete disfunção orgânica, que pode ser múltiplas, com graves alterações no metabolismo tecidual secundário ao distúrbio circulatório, colocando a vida do indivíduo em risco. ${ }^{2}$

A definição anterior de sepse considerava marcadores inflamatórios tradicionais, como alteração do leucograma, febre ou hipotermia, taquicardia e taquipneia, como critérios para diagnosticar sepse. ' Quando havia disfunção orgânica associada a dois ou mais desses marcadores, o termo "sepse grave" era empregado e uma série de medidas terapêuticas eram instituídas (e.g., expansão volêmica, antibióticos, etc.). Esses critérios eram pouco intuitivos para a comunidade médica e apresentavam baixa acurácia para desfechos clínicos relevantes, como a morte.

Alteração nos marcadores de resposta inflamatória da SIRS (systemic inflammatory response syndrome), frequentemente, representam uma resposta fisiológica à infecção, mas não apenas por essa condição clínica, o que pode ser uma explicação para essa baixa acurácia. Assim, em 2016, um novo consenso internacional foi estabelecido (Sepsis-3). ${ }^{1.3}$ Dessa forma, variáveis de fácil aplicação e com maior acurácia na predição de morte em pacientes infectados foram escolhidas. A variável que melhor preencheu esses critérios foi a elevação do escore em pelo menos dois pontos no Sequential Organ Failure Assessment. Além disso, especialmente em ambientes fora da unidade de terapia intensiva (UTI), uma versão simplificada do SOFA (Quick SOFA, qSOFA) pode ser aplicada e, dessa maneira, a sepse pode ser presumida, antes mesmo de que os resultados de exames laboratoriais estejam disponíveis. Esses novos critérios auxiliam a estratificar a gravidade e a direcionar a atenção e os recursos a pacientes mais graves e, por isso, com uma maior chance de morte.

Os benefícios do uso de um protocolo clínico relacionado com sepse são enumerados na Figura 1.
Redução da letalidade

Redução no tempo de internação hospitalar

Redução nos custos do tratamento

Retorno precoce do paciente a suas atividades habituais

Diferencial na qualidade do atendimento multiprofissional

Figura 1. Benefícios possíveis do uso, como rotina, de um protocolo clínico relacionado com sepse.

O objetivo dessa revisão foi estabelecer um protocolo clínico de apoio à decisão, idealmente registrado no prontuário eletrônico com triagem, deflagrando o início imediato do tratamento para sepse, com condutas baseadas em evidências científicas padronizadas por comissão técnica e de gestores, com o intuito de garantir boas práticas assistenciais.

\section{Métodos}

Esse estudo é uma revisão narrativa, usando como referência - Protocolo Clínico de sepse elaborado pela Unimed Recife. Vários estudos foram consultados para se elaborar o Protocolo Clínico de sepse da Unimed Recife. 1, 4-10

\section{Definições de sepse e choque séptico}

Define-se sepse como uma disfunção orgânica potencialmente fatal, causada por uma resposta imune desregulada deflagrada por uma infecção. Por sua vez, choque séptico é definido como presença de hipotensão arterial mantida, que tem o potencial de aumentar a letalidade substancialmente, quando há necessidade de uso de vasopressores para se manter uma pressão arterial média (PAM) $\geq 65 \mathrm{mmHg}$ associada a lactato $\geq 2 \mathrm{mmol} / \mathrm{L}$, após adequada ressuscitação volêmica (Figura 2).

\section{Considerações}

Taxas de letalidade hospitalar por sepse são superiores a $40 \%$. A inexistência de marcador biológico e a heterogeneidade do que constitui suspeita de infecção trazem limitações nos estudos em sepse. As definições de sepse e choque séptico foram revisadas, considerando avanços recentes no entendimento da fisiopatologia, manejo e epidemiologia da sepse. Em 2016, uma força tarefa foi convocada pela Society of Critical Care Medicine e pela European Society of Intensive Care Medicine. Durante várias reuniões, essa força tarefa estabeleceu definições e critérios clínicos, após utilização do método Delphi, análise de bases de dados de registros eletrônicos de saúde e votação, seguidos de circulação para sociedades profissionais internacionais, solicitando revisão por pares e, finalmente, o endosso por 31 sociedades. Dessa forma, houve a publicação da revisão clínica do Terceiro Consenso Internacional Sobre Sepse e Choque Séptico, surgindo, assim, novas definições. ${ }^{1}$ 


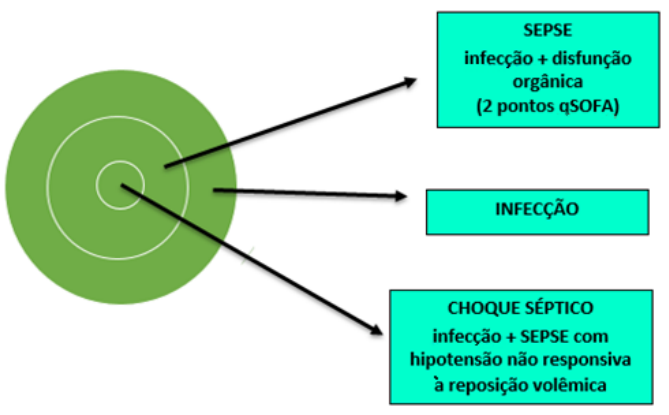

Figura 2. Critérios clínicos de sepse e choque séptico.

Pontos importantes sobre sepse:

- Sepse é definida como uma disfunção orgânica potencialmente fatal, causada por uma resposta desregulada do hospedeiro à infecção;

- Sepse atual é igual a sepse grave anterior;

- Choque séptico é quando, após adequada expansão volêmica, o hospedeiro persiste com alteração circulatória, celular ou metabólica;

- Pacientes com choque séptico podem ser clinicamente identificados por uma exigência de droga vasoativa para manter uma pressão arterial média de $65 \mathrm{mmHg}$, ou maior, e nível de lactato sérico maior que $2 \mathrm{mmol} / \mathrm{L}$ (>18 mg/dl) na ausência de hipovolemia. Por outro lado, em ambientes extra-hospitalares, emergências ou enfermarias gerais, os pacientes adultos, com suspeita de infecção, podem ser rapidamente identificados como mais propensos a terem resultados ruins, típicos da sepse, se preencherem, pelo menos, dois dos seguintes critérios clínicos que, juntos, constituem um novo escore clínico à beira do leito denominado qSOFA: frequência respiratória de 22/minuto ou superior, alteração do nível de consciência ou pressão arterial sistólica de 100 mmHg ou menos. Essas definições e critérios clínicos alterados substituem as definições anteriores, em tese, oferecendo maior consistência para estudos epidemiológicos e ensaios clínicos, e facilitando o reconhecimento precoce e o manejo mais oportuno de pacientes com sepse ou em risco de desenvolver sepse. Nesse protocolo, optamos por seguir as definições pelas necessidades e estrutura da instituição.

Como se trata de uma disfunção orgânica secundária a uma resposta desregulada do hospedeiro a um agente infeccioso, quando possível usaremos o escore de SOFA, para sua identificação.

O qSOFA é uma novidade e serve como rastreio para pacientes fora do ambiente da UTI, com risco de desenvolver sepse. A grande utilidade do qSOFA é que, ao contrário do escore matriz (SOFA), não usa qualquer variável laboratorial, tornando-o muito fácil de se usar à beira do leito. Se pelo menos 2 das 3 variáveis forem encontradas, recomenda-se investigar disfunção orgânica pelo SOFA, reavaliar a terapia, aumentar a monitorização e considerar referenciar o paciente a um especialista em Medicina Intensiva. Portanto, em um paciente sem suspeita de infecção, um qSOFA positivo deve levantar a hipótese de infecção.

Os critérios usados são:

- Pressão arterial sistólica menor que 100 mmHg;

- Frequência respiratória maior que $22 / \mathrm{min}$;

- $\quad$ Alteração do estado mental (GCS < 15).

Cada variável conta um ponto no escore, portanto, ele vai de 0 a 3. Uma pontuação igual ou maior a 2 indica maior risco de morte ou de permanência prolongada na UTI.

Dessa forma, sepse é agora, clinicamente definida, como quadro infeccioso associado à disfunção orgânica. Essa disfunção orgânica é identificada pelo escore do SOFA. Uma variação aguda, de 2 pontos ou mais no SOFA, associada à infecção define o estado séptico.

Alguns parâmetros são considerados para determinar a disfunção orgânica, como: (1) PAM $<70$ mmHg; (2) rebaixamento do nível de consciência/agitação; (3) diurese $<0,5$ $\mathrm{ml} / \mathrm{Kg} / \mathrm{h}$ ou creatinina $\geq 1,2 \mathrm{mg} / \mathrm{dl}$; (4) Elevação de bilirrubinas; (5) Plaquetopenia $<150.000 \mathrm{~mm}^{3}$ e (6) relação PO2/ $\mathrm{FiO}^{2}<400$.

\section{Conduta}

As medidas iniciais que deverão ser realizadas na primeira hora, incluem:

\section{Ressuscitação volêmica e otimização da perfusão}

1. Recomenda-se administrar, pelo menos, $30 \mathrm{ml} / \mathrm{kg}$ de cristaloides, nas primeiras 3 horas.

2. Após essa fase inicial de ressuscitação volêmica, os volumes adicionais deverão ser guiados por reavaliações frequentes (ou seja, avaliar fluido responsividade) necessidade). Esse ponto será abordado agora, pela importância da ressuscitação e pelos efeitos deletérios da reposição hídrica exagerada.

\section{Fluido responsividade}

- A conduta de Fluido responsividade é definida como aumento de $10-15 \%$ no débito cardíaco ou no volume sistólico, em resposta a um desafio ao sistema cardiovascular. Esse desafio pode ser realizado com a prova de volume, obtendo-se a monitorização do paciente antes 
e após a infusão de líquidos (exemplo, cristaloides -500 $\mathrm{ml}$ ), em um determinado período de tempo (10 minutos);

- Só haverá fluido responsividade, quando o volume oferecido promover um acréscimo na volemia e, por conseguinte, na pré-carga, aumentando a quantidade de sangue ventricular no fim da diástole e, assim, aumentando o débito cardíaco. Vale salientar que esse aumento do débito cardíaco secundário ao aumento da volemia, só acontece até um determinado ponto. A partir desse ponto, todo volume ofertado não acarretará em aumento do débito cardíaco, mas ao contrário, trará riscos ao paciente, com aumento das pressões hidrostáticas e edema pulmonar e sistêmico. Portanto, o paciente que não for fluido responsivo, não deve receber infusão de volume.

A fluido-responsividade pode ser aferida de forma mais eficaz pela medida dinâmica do débito cardíaco, por meio do cateter de artéria pulmonar ou pelo ecocardiograma. Outra forma inclui a variação da pressão de pulso. $\bigcirc$ diâmetro da veia cava pode ser um indicador de hipovolemia.

Embora sinais clínicos, como hipotensão, taquicardia, perfusão periférica lentificada, tempo de enchimento capilar superior a três segundos e extremidades frias, sejam importantes para identificar situações de perfusão inadequada, esses dados não têm o potencial de determinar volemia e responsividade a fluidos.

A pressão venosa central (PVC) ou a sua variação também não apresenta acurácia adequada. Da mesma forma o aumento da pressão arterial média (PAM) após a expansão é um pobre preditor de responsividade. Atualmente, a utilização do ECOTT na beira do leito para a realização do VTI, com a finalidade de estimar o débito cardíaco após expansão volêmica, vem sendo realizada com maior frequência.

Outro ponto importante é que o fato de ser fluido responsivo não significa que o paciente necessite de volume. Pessoas saudáveis são fluido responsivas, porém não necessitam de infusão de volume. Essa fluido-necessidade está presente nos pacientes com evidência clínico-laboratorial de hipóxia tissular e hipoperfusão tecidual, tais como: hiperlactatemia, baixa saturação venosa central ou mista de $\mathrm{O}_{2}$, hipotensão, taquicardia, oligúria e rebaixamento do nível de consciência. De forma simples, haverá fluido-responsividade pela aferição de aumento no débito cardíaco e fluido-necessidade, quando houver evidência de hipoperfusão tissular: hiperlactatemia e baixa saturação venosa central de $\mathrm{O}_{2}$.

Dessa forma, após evidência da fluido-responsividade, estabeleceremos metas para reposição de volume, monitorização hemodinâmica e recuperação da perfusão tecidual, como:

- $\quad P A M \geq 70 \mathrm{mmHg}$;

- $\quad$ PVC 8-12 mmHg (ou 15, nos pacientes em ventilação);
- $\quad$ Diurese $\geq 0,5 \mathrm{ml} / \mathrm{Kg} / \mathrm{hora}$;

- Clareamento e normalização do lactato;

- Saturação venosa central $>70 \%$ ou saturação venoso misto > 65\%;

- $\quad$ GAP pCO2 <6.

É importante ressaltar que o volume ofertado, para que essas metas sejam alcançadas, gere, obrigatoriamente, uma resposta positiva no débito cardíaco. Quando não mais isso acontecer, deve-se otimizar as metas de perfusão tissular com outras medidas, tais como dobutamina, vasopressores e hemoderivados.

Abaixo, são citados alguns parâmetros e testes que podem sugerir o estado volêmico do paciente:

1. Parâmetros clínicos de hipervolemia (crepitações, estase jugular, dispneia, edema e hipertensão) ou de hipovolemia (hipotensão arterial, rebaixamento de consciência, taquicardia, oligúria, desidratação e tempo de enchimento capilar maior que 3 segundos).

2. Parâmetros laboratoriais de hipovolemia (hipernatremia, alcalose metabólica, hiperlactatemia, baixa saturação venosa central e gap pCO2 elevado).

A administração de fluidos é um dos aspectos principais no manejo inicial do paciente séptico, porém a restauração da volemia e da perfusão tecidual permanece um desafio, pois baseia-se não só na experiência do médico, como também nas necessidades individuais de cada paciente e nas limitações de cada método.

\section{Otimização hemodinâmica Uso de vasopressores}

1. Tratamento com vasopressores tem como meta inicial uma PAM de $65 \mathrm{mmHg}$;

2. Norepinefrina como primeira opção de vasopressor. A noradrenalina (por efeito alfa-1 e beta-1) pode gerar aumento de PAM, aumento do débito cardíaco e meIhora do retorno venoso, sem comprometer o balanço hídrico do paciente;

3. Sugere-se adição de epinefrina ou vasopressina quando for necessário um segundo agente para manter a pressão arterial adequada;

4. Vasopressina (até 0,03 unidades/minuto) pode ser adicionada à norepinefrina, com o objetivo de se elevar a PAM ou, alternativamente, diminuir a dosagem de norepinefrina;

5. Dopamina como agente vasopressor alternativo à norepinefrina somente deve ser usada em pacientes altamente selecionados (exemplo, pacientes com baixo risco de taquiarritmias e bradicardia relativa ou absoluta);

6. Todos os pacientes que precisem de vasopressores devem receber um cateter arterial o mais cedo possível, no caso de haver recursos disponíveis. 


\section{Otimização da perfusão tecidual}

Procura-se, inicialmente, obter as metas de saturação venosa central e de lactato com a infusão de volume.

Caso o paciente persista com baixa saturação venosa de 02, apesar de já haver sido realizada uma ressuscitação hídrica otimizada (ou seja, não é mais fluido responsivo), poderemos lançar mão de duas alternativas: para aqueles com hemoglobina $<7 \mathrm{~g} / \mathrm{dl}$, faremos concentrado de hemácias, e para os demais pacientes iniciaremos um inotrópico, e.g., dobutamina. Como descrito no item anterior, para aqueles com PAM baixa, que não responda à infusão de líquidos, faremos vasopressores.

\section{Otimização antimicrobiana e controle do foco}

Antibioticoterapia de amplo espectro de acordo com o foco provável e protocolo institucional, podendo ser alterado conforme outros critérios clínicos e laboratoriais observados. Recomendando-se reavaliação e ajuste conforme resultados de culturas nos primeiros dias e se não for observada uma melhora clínica. Nessa situação, a dosagem de procalcitonina pode ser benéfica em sua decisão. $O$ tempo de manutenção do antibiótico, normalmente, será de 5 a 10 dias, tendo como guia para suspensão a resposta clínica.

\section{Foco infeccioso}

Os princípios de controle, no tratamento de sepse, incluem também o controle eficiente do foco de infecção, como no exemplo de drenagem de um abcesso, desbridamento de tecido necrosado e infectado, remoção de um dispositivo potencialmente infectado e o controle definitivo de uma fonte de contaminação microbiana contínua.

\section{Atenção aos exames complementares}

Devemos ter atenção aos exames complementares para essa investigação metódica, que, quando realizados, podem ser utilizados também na avaliação prognóstica, tais como:

- $\quad$ ECG;

- Exames de imagem

- Hemograma;

- Glicemia, ureia, creatinina, ionograma, cálcio, fósforo, magnésio, CPK, ckmb, troponina, INR, gasimetria arterial, gasometria venosa central, TSH, Procalcitonina, PCR, $\mathrm{BT}, \mathrm{BD}, \mathrm{BI}, \mathrm{FA}, \mathrm{TGO}, \mathrm{TGP}$, sumário de urina;

- Hemoculturas (duas) e culturas de sítios pertinentes, conforme possibilidade de foco (ainda na primeira hora, de sítios diferentes, antes do antimicrobiano, sem, contudo, atrasá-lo);

- Ecocardiograma.

\section{Medidas adicionais Corticoide}

Nos pacientes com choque refratário à reposição hídrica e ao uso de vasopressores, sugere-se hidrocortisona $200 \mathrm{mg}$ intravenosa em 24 horas.

\section{Produtos sanguíneos}

Recomenda-se que a transfusão de concentrado de hemácias ocorra apenas quando a hemoglobina for menor que $7,0 \mathrm{~g} /$ dl em adultos, na ausência de circunstâncias como isquemia miocárdica, hipoxemia grave ou hemorragia aguda. Essa é uma sugestão contrária ao uso de plasma fresco congelado para corrigir coagulopatias na ausência de sangramento ou programação de procedimentos invasivos.

Sugere-se a transfusão profilática de concentrado de plaquetas quando plaquetas $<10.000 / \mathrm{mm}^{3}$ na ausência de sangramento aparente e quando $<20.000 / \mathrm{mm}^{3}$ nos pacientes com significativo risco de sangramento. Valores maiores de plaquetas ( $\geq 50.000 / \mathrm{mm} 3$ ) uma transfusão é recomendada em casos de sangramento ativo, cirurgia ou procedimentos invasivos.

\section{Ventilação mecânica}

Recomenda-se utilizar volume corrente de $6 \mathrm{~mL} / \mathrm{Kg}$ de peso corporal predito. Recomenda-se a utilizar pressão de platô de até $30 \mathrm{cmH}_{2} \mathrm{O}$. Sugere-se utilizar valores altos de pressão respiratória final positiva (PEEP). Sugere-se realizar manobras de recrutamento em adultos com SDRA grave induzida pela sepse. Recomenda-se utilizar posição prona ao invés de supina em pacientes adultos com SDRA induzida pela sepse e $\mathrm{PaO}_{2} / \mathrm{FiO}_{2}<150$. Sugere-se utilizar bloqueador neuromuscular por $\leq 48$ horas em pacientes com SDRA induzida pela sepse e $\mathrm{PaO}_{2} / \mathrm{FiO}_{2}<150$. Recomendação contrária ao uso de beta-2 agonista para o tratamento de pacientes sem broncoespasmo ativo e com SDRA induzida pela sepse. Recomenda-se manter a cabeceira elevada a $30-45^{\circ}$ em pacientes sépticos em ventilação mecânica (VM) a fim de reduzir o risco de aspirações e evitar pneumonia associada à VM.

\section{Sedação e analgesia}

Recomenda-se que a sedação contínua ou intermitente seja minimizada em pacientes com sepse em VM, visando à titulação com objetivos específicos, como avaliar a retirada da sedação e testar a retirada de VM.

\section{Controle glicêmico}

Recomenda-se iniciar doses de insulina quando dois valores consecutivos de glicemia forem $>180 \mathrm{mg} / \mathrm{dl}$. Essa abordagem deve visar a um limite superior de glicemia $<180 \mathrm{mg} / \mathrm{ml}$.

\section{Profilaxia de Trombose Venosa Profunda}

Recomenda-se profilaxia farmacológica (heparinas de baixo peso molecular, HBPM, preferencialmente, ao uso de heparina não fracionada, HNF) para profilaxia de trombose venosa profunda (TVP), na ausência de contraindicações ao uso desses agentes. Sugere-se profilaxia farmacológica associada à profilaxia mecânica de TVP (sempre que possível afastada a presença de trombose venosa periférica). 


\section{Profilaxia de úlcera de estresse}

Recomenda-se que a profilaxia de úlcera de estresse seja oferecida para pacientes com sepse ou choque séptico com fatores de risco para sangramento gastrointestinal (e.g., coagulopatia, VM por pelo menos 48 horas, possivelmente, hipotensão arterial), utilizando inibidores da bomba de prótons ou antagonistas do receptor da $\mathrm{H}_{2}$.

\section{Nutrição}

Sugere-se: (1) dieta enteral trófica/hipocalórica ou plena, com início precoce em pacientes críticos com sepse ou choque séptico, ao invés de jejum ou apenas soro glicosado IV; (2) uso de procinéticos em pacientes críticos com sepse ou choque séptico e intolerância à dieta; ou, (3) sondagem pós-pilórica em pacientes críticos com sepse ou choque séptico com intolerância à dieta ou considerados de alto risco para aspiração.

\section{Indicadores de qualidade}

Os protocolos gerenciados buscam, por meio da instituição de diretrizes assistenciais e da monitorização contínua de indicadores de qualidade, garantir a implementação de ações específicas, baseada na melhor evidência científica possível.

Indicadores de qualidade, a serem utilizados no protocolo, são mostrados nas Tabelas 2 e 3.

Tabela 1. Lista de indicadores de qualidade que devem ser observados no tratamento de pacientes com sepse que precisam ser gerenciados no Protocolo de Sepse

\begin{tabular}{|c|c|c|}
\hline \multicolumn{3}{|c|}{ BÁSICOS } \\
\hline Indicador & Descrição & Formula \\
\hline Aderência global & $\begin{array}{l}\text { Ter sido aderente a todos } \\
\text { os itens pertinentes acima } \\
\text { mencionados }\end{array}$ & $\begin{array}{l}\text { - Numerador: número de pacientes } \\
\text { aderentes a todos indicadores } \\
\text { pertinentes; } \\
\text { - Denominador: todos os pacientes } \\
\text { com sepse/choque séptico. }\end{array}$ \\
\hline Lactato & $\begin{array}{l}\text { Coleta de lactato dentro da } \\
\text { primeira hora }\end{array}$ & $\begin{array}{l}\text { - Numerador: número de pacientes } \\
\text { que coletaram lactato dentro da } \\
\text { primeira hora do diagnóstico da sepse; } \\
\text { - Denominador: todos os pacientes } \\
\text { com sepse/choque séptico. }\end{array}$ \\
\hline Hemoculturas & $\begin{array}{l}\text { Coleta de hemocultura antes } \\
\text { do início do antimicrobiano }\end{array}$ & $\begin{array}{l}\text { - Numerador: número de pacientes } \\
\text { que coletaram hemocultura antes do } \\
\text { início antimicrobiano e dentro da } \\
\text { primeira hora do diagnóstico da sepse } \\
\text { - Denominador: todos os pacientes } \\
\text { com sepse/choque séptico }\end{array}$ \\
\hline Antimicrobiano & $\begin{array}{l}\text { Administração endovenosa } \\
\text { de antimicrobianos de } \\
\text { espectro adequado, em } \\
\text { tempo adequado }\end{array}$ & $\begin{array}{l}\text { - Numerador: número de pacientes } \\
\text { em que o início da administração do } \\
\text { antimicrobiano de espectro adequado } \\
\text { ocorreu dentro da primeira hora do } \\
\text { diagnóstico da sepse; } \\
\text { - Denominador: todos os pacientes } \\
\text { com sepse/choque séptico. }\end{array}$ \\
\hline $\begin{array}{l}\text { Letalidade } \\
\text { hospitalar }\end{array}$ & $\begin{array}{l}\text { Óbito durante a internação } \\
\text { hospitalar }\end{array}$ & $\begin{array}{l}\text { - Numerador: pacientes com óbito } \\
\text { durante a internação hospitalar } \\
\text { - Denominador: todos os pacientes } \\
\text { com sepse/choque séptico internados }\end{array}$ \\
\hline
\end{tabular}

Tabela 2. Indicadores monitorados do Protocolo de Sepse

\begin{tabular}{|c|c|c|}
\hline \multicolumn{3}{|c|}{ DESEJÁVEIS } \\
\hline Indicador & Descrição & Formula \\
\hline $\begin{array}{l}\text { Tempo de } \\
\text { disfunção } \\
\text { orgânica }\end{array}$ & $\begin{array}{l}\text { Tempo decorrido entre a } \\
\text { instalação da primeira } \\
\text { disfunção orgânica e a } \\
\text { formulação da hipótese } \\
\text { diagnóstica de sepse }\end{array}$ & $\begin{array}{l}\text { - Numerador: soma do tempo } \\
\text { para diagnóstico da disfunção nos } \\
\text { pacientes sepse/choque séptico; } \\
\text { - Denominador: número total de } \\
\text { pacientes com sepse/choque séptico; } \\
\text { - Resultado expresso em média } \pm \\
\text { desvio padrão. }\end{array}$ \\
\hline $\begin{array}{l}\text { Tempo para } \\
\text { terapia } \\
\text { antimicrobiana }\end{array}$ & $\begin{array}{l}\text { Tempo decorrido entre a } \\
\text { formulação da hipótese } \\
\text { diagnóstica de sepse e o } \\
\text { início da infusão do primeiro } \\
\text { antimicrobiano }\end{array}$ & $\begin{array}{l}\text { - Numerador: soma dos tempos para } \\
\text { início de antimicrobiano nos pacientes } \\
\text { com sepse/choque séptico; } \\
\text { - Denominador: número total } \\
\text { de pacientes com sepse/choque } \\
\text { séptico. Pacientes já em uso de } \\
\text { antimicrobianos e que não tiveram } \\
\text { seu esquema alterado não são } \\
\text { considerados; } \\
\text { - Resultado expresso em média } \pm \\
\text { desvio padrão. }\end{array}$ \\
\hline $\begin{array}{l}\text { Reposição } \\
\text { volêmica }\end{array}$ & $\begin{array}{l}\text { Início de infusão de } 30 \mathrm{ml} / \\
\mathrm{kg} \text { na primeira hora após o } \\
\text { início da hipotensão ou do } \\
\text { momento de coleta do lactato } \\
\text { se esse estiver acima de duas } \\
\text { vezes o valor de referência }\end{array}$ & $\begin{array}{l}\text { - Numerador: pacientes que } \\
\text { iniciaram a reposição volêmica } \\
\text { em até uma hora após o início da } \\
\text { hipotensão ou da coleta de lactato e } \\
\text { receberam pelo menos } 30 \mathrm{ml} / \mathrm{Kg} \text { de } \\
\text { cristaloide (ou menores quantidades } \\
\text { se justificando em prontuário); } \\
\text { - Denominador: pacientes com } \\
\text { lactatos acima de duas vezes o valor } \\
\text { de referência ou com pressão arterial } \\
\text { média abaixo de } 65 \mathrm{mmHg} \text {. }\end{array}$ \\
\hline Vasopressor & $\begin{array}{l}\text { Uso de vasopressores em } \\
\text { pacientes que permanecem } \\
\text { hipotensos após reposição } \\
\text { volêmica }\end{array}$ & $\begin{array}{l}\text { - Numerador: pacientes que } \\
\text { iniciaram a reposição volêmica e que } \\
\text { receberam vasopressores para manter } \\
\text { a pressão arterial média acima de } \\
65 \mathrm{mmHg} \text { em até uma hora do início } \\
\text { da disfunção; } \\
\text { - Denominador: pacientes que } \\
\text { permaneceram com pressão arterial } \\
\text { média abaixo de } 65 \mathrm{mmHg} \text { após } \\
\text { volume. }\end{array}$ \\
\hline $\begin{array}{c}\text { Coleta de } 2^{\circ} \\
\text { lactato }\end{array}$ & $\begin{array}{l}\text { Coleta de segunda amostra } \\
\text { de lactato até a } 4^{\circ} \text { hora após } \\
\text { o diagnóstico da sepse em } \\
\text { pacientes cujo lactato inicial } \\
\text { estava acima de } 2 \text { vezes o } \\
\text { valor de referência }\end{array}$ & $\begin{array}{l}\text { - Numerador: pacientes submetidos } \\
\text { à segunda coleta de lactato dentro } \\
\text { de } 4 \text { horas do diagnóstico da sepse; } \\
\text { - Denominador: pacientes com } \\
\text { lactato inicial acima de } 2 \text { vezes o } \\
\text { valor normal. }\end{array}$ \\
\hline $\begin{array}{c}\text { Reavaliação } \\
\text { da volemia e } \\
\text { perfusão }\end{array}$ & $\begin{array}{l}\text { Reavaliação de sinais vitais, } \\
\text { parâmetros de perfusão e } \\
\text { de volemia nos pacientes } \\
\text { pertinentes em até } 6 \text { horas } \\
\text { após o diagnóstico da sepse }\end{array}$ & $\begin{array}{l}\text { - Numerador: pacientes em que há } \\
\text { registro de reavaliação de volemia } \\
\text { e perfusão pelo médico dentro de } 6 \\
\text { horas do diagnóstico de sepse; } \\
\text { - Denominador: pacientes que } \\
\text { necessitaram de vasopressores para } \\
\text { manter a pressão arterial média } \\
\text { acima de } 65 \mathrm{mmHg} \text {, após receber } \\
\text { volume ou cujo lactato inicial era } \\
\text { acima de } 2 \text { vezes o valor normal. }\end{array}$ \\
\hline
\end{tabular}

\section{Conclusão}

A revisão, que descrevemos nesse artigo, foi baseada em evidências científicas atualizadas para o protocolo vigente, como forma de alinhar com a maioria dos serviços hospitalares. Os indicadores propostos, os básicos e expandidos, permanecerão monitorados, avaliados e divulgados para a equipe, tanto como continuaremos com treinamento contínuo dos atores envolvidos na atualização do Protocolo de Sepse. 


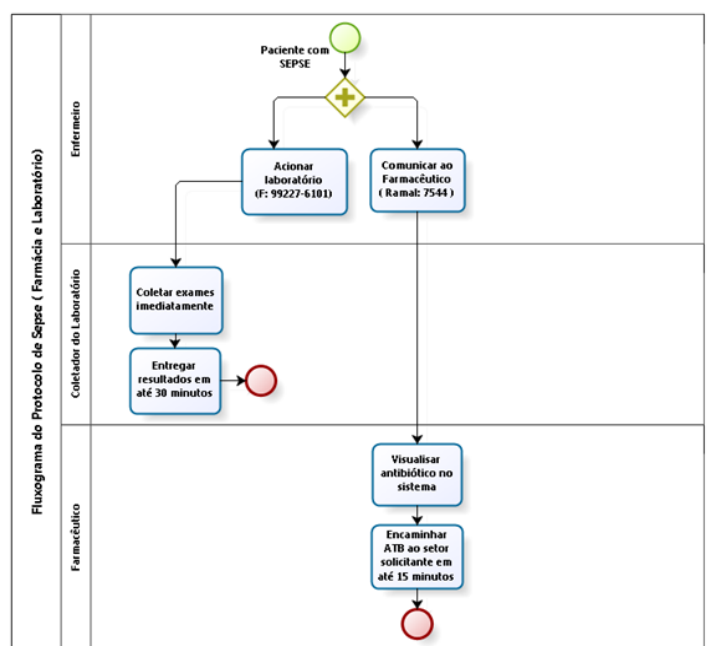

Figura 2. Fluxograma do processo de acionamento dos setores de farmácia e laboratório.

Contribuição dos autores Todos os autores deste artigo contribuíram na elaboração do Protocolo Clínico usado na Unimed Recife; Revisão final do texto, CAB e MMRFF.

Financiamento Não houve financiamento para este estudo.

Conflito de interesse Os autores são funcionários da Unimed Recife.

Cátia Arcuri Branco

https://orcid.org/0000-0002-7082-9223

Jackeline Soares Costa

https://orcid.org/0000-0003-1520-0454

Girlayne Batista Arruda

https://orcid.org/0000-0002-2723-7185

Willyane Gracy Aguiar de Andrade Gomes de Souza

https://orcid.org/0000-0002-1985-1732

Alex Maurício Garcia Santos

https://orcid.org/ 0000-0002-7472-041X

Fabiana Gonzaga Torreão dos Santos

https://orcid.org/0000-0002-5913-1297

Jeniffer Cristina Rocha Cruz

https://orcid.org/0000-0002-3533-177X

Lais Emanoelle de Souza Cabral

https://orcid.org/0000-0001-7891-0395

Adriana Cesário Lopes

https://orcid.org/0000-0002- 3555-9221

Orlando José de Lima Nascimento

https://orcid.org/0000-0002-4531-1078

Martha Maria Romeiro Figueiroa F. Fonseca

https://orcid.org/0000-0003-4929-6343

\section{Referências}

1. Levy MM, Fink MP, Marshall JC, Abraham E, Angus D, Cook D, Cohen J, Opal SM, Vincent JL, Ramsay G; International Sepsis Definitions Conference. 2001 SCCM/ ESICM/ACCP/ATS/SIS International Sepsis Definitions Conference. Intensive Care Med. 2003 Apr;29(4):530-8. doi: 10.1007/s00134-003-1662-x. Epub 2003 Mar
28. PMID: 12664219.

2. Charitos IA, Topi S, Castellaneta F, D'Agostino D. Current Issues and Perspectives in Patients with Possible Sepsis at Emergency Departments. Antibiotics. 2019; 8(2):56. https://doi.org/10.3390/antibiotics8020056

3. Singer $M$, Deutschman CS, Seymour CW, et al. The Third International Consensus Definitions for Sepsis and Septic Shock (Sepsis-3). JAMA. 2016;315:801-810.

4. Shankar-Hari M, Phillips GS, Levy ML, et al. Developing a New Definition and Assessing New Clinical Criteria for Septic Shock: For the Third International Consensus Definitions for Sepsis and Septic Shock (Sepsis-3). JAMA. 2016;315:775-787.

5. Seymour CW, Liu VX, Iwashyna TJ, et al. Assessment of Clinical Criteria for Sepsis: For the Third International Consensus Definitions for Sepsis and Septic Shock (Sepsis-3). JAMA. 2016;315:762-774.

6. Churpek MM, Snyder A, Sokol S, Pettit NN, Edelson DP. Investigating the Impact of Different Suspicion of Infection Criteria on the Accuracy of Quick Sepsis-Related Organ Failure Assessment, Systemic Inflammatory Response Syndrome, and Early Warning Scores. Crit Care Med. 2017;45:1805-1812.

7. Dellinger RP, Levy MM, Rhodes A, et al. Surviving sepsis campaign: international guidelines for management of severe sepsis and septic shock: 2012. Crit Care Med. 2013;41:580-637.

8. Levy MM, Fink MP, Marshall JC, et al. 2001 SCCM/ ESICM/ACCP/ATS/SIS International Sepsis Definitions Conference. Intensive Care Med. 2003;29:530-538.

9. Sepse ILAd. Roteiro de Implementação de Protocolo Assistencial Diferenciado: campanha de sobrevivência a Sepse. In. Vila Clementino - SP: Instituto Latino Americano de Sepse 2019, pp. 39.

10. Vincent JL, de Mendonca A, Cantraine F, et al. Use of the SOFA score to assess the incidence of organ dysfunction/failure in intensive care units: results of a multicenter, prospective study. Working group on "sepsis-related problems" of the European Society of Intensive Care Medicine. Crit Care Med. 1998;26:1793-1800.

11. Vincent JL, Moreno R, Takala J, et al. The SOFA (Sepsis-related Organ Failure Assessment) score to describe organ dysfunction/failure. On behalf of the Working Group on Sepsis-Related Problems of the European Society of Intensive Care Medicine. Intensive Care Med. 1996;22:707-710.

12. Vincent JL, Opal SM, Marshall JC, Tracey KJ. Sepsis definitions: time for change. Lancet. 2013;381:774-775.

13. Shankar-Hari, M., Ambler, M., Mahalingasivam, V. et al. Evidence for a causal link between sepsis and long-term mortality: a systematic review of epidemiologic studies. Crit Care 20, 101 (2016). https://doi.org/10.1186/ s13054-016-1276-7

14. Panita W. and Prasit W. In Septic shock in the ER: diagnostic and management challenges: Open Access Emerg Med. 2019; 11: 77-86. Published online 2019 Apr 11. doi: 10.2147/OAEM.S166086 